\title{
Nuevos instrumentos de financiación para el sector no lucrativo: el reto del crowdfunding social
}

Antonia Sajardo-Moreno*

Doctora en Ciencias Económicas. Profesora Titular, Departamento de Economía Aplicada, Instituto Universitario Polibienestar, Universitat de València, Valencia, España. Correo electrónico:

antonia.sajardo@uv.es

Recibido: 11 de mayo del 2015 Aceptado: 9 de noviembre del 2015

Cómo citar este artículo: Sajardo-Moreno, A. (2016). Nuevos instrumentos de financiación para el sector no lucrativo: El reto del crowdfunding social. Cooperativismo y Desarrollo, 24(108), 39-53. doi: http://dx.doi. org/10.16925/co.v24i108.1259

\section{Resumen}

Propósito: exponer la manera en que la crisis económica en España ha venido haciendo especialmente notoria la debilidad financiera del sector no lucrativo, tradicionalmente dependiente del apoyo público. El agotamiento de las arcas públicas, el receso en las donaciones privadas y el incremento de la reticencia del recurso financiero por la banca tradicional sitúan al sector ante la disyuntiva de un progresivo deterioro como fuerza económica y social. Descripción: en este contexto, el sector no lucrativo español se ha visto impelido a explorar nuevas formas de financiación, tornando una nueva mirada hacia su mismo origen; hacia su base social: la ciudadanía. Punto de vista: es de destacar que la ciudadanía, en este siglo, se halla inmersa en un nuevo paradigma tecnológico: el paradigma digital, el cual viene a conformarse como el instrumento de primer orden para una nueva forma de solidaridad social. Conclusiones: uno de los principales exponentes de la nueva solidaridad social lo configura el crowdfunding o financiación en masa a través de Internet, de cuyo análisis y potencialidades se ocupa este trabajo.

Palabras clave: crowdfunding, crowdfunding social, economía social, fundraising, sector no lucrativo. 


\title{
New Financing Instruments for the Non-Profit Sector: The Challenge of Social Crowdfunding
}

\begin{abstract}
Purpose: To explain how the economic crisis in Spain has especially made known the financial weakness of the non-profit sector, traditionally dependent on public support. Depletion of public funds, recess in private donations and increased reticence of financial resources by traditional banking put the sector in the dilemma of progressive deterioration as economic and social force. Description: In this context, the Spanish non-profit sector has been driven to explore new forms of financing, giving a fresh look to its origin itself, to its social base: citizenship. Point of view: It is noteworthy that citizenship, in this century, is immersed in a new technological paradigm: the digital paradigm, which became the major instrument of a new form of social solidarity. Conclusions: One of the leading exponents of the new social solidarity is crowdfunding, whose analysis and potentials are dealt with in this work.
\end{abstract}

Keywords: crowdfunding, social crowdfunding, social economy, fundraising, non-profit sector.

\section{Novos instrumentos de financiamento para o setor sem fins lucrativos: o desafio do crowdfunding social}

\section{Resumo}

Propósito: expor como a crise econômica na Espanha vem tornando evidente a debilidade financeira do setor sem fins lucrativos, tradicionalmente dependente do apoio público. O esgotamento dos cofres públicos, a redução das doações privadas e o aumento da reticência do recurso financeiro pela banca tradicional posicionam o setor ante a disjuntiva de uma progressiva deterioração como força econômica e social. Descrição: nesse contexto, o setor sem fins lucrativos espanhol tem sido visto impelido a explorar novas formas de financiamento, colocando um novo olhar em sua mesma origem; em sua base social: a cidadania. Ponto de vista: é de destacar que a cidadania, neste século, encontra-se imersa num novo paradigma tecnológico: o digital, que vem conformando-se como o instrumento de primeira ordem para uma nova forma de solidariedade social. Conclusões: o crowdfunding ou o financiamento em massa por meio da Internet se configura como um dos principais expoentes da nova solidariedade social, de cuja análise e potencialidades este trabalho se trata.

Palavras-chave: crowdfunding, crowdfunding social, economia social, fundraising, setor sem fins lucrativos. 


\section{Introducción}

La variable financiera ha sido siempre un tema de crucial importancia en el seno de las entidades del sector no lucrativo. Su alta dependencia financiera del sector público (ostensiblemente superior al 50\%; Sajardo, Serra y Yodi, 2007), la naturaleza voluntaria de sus aportes, unida a su gran atomización, así como el hecho de hallarse muy alejadas de los planteamientos (misión-visión) de las entidades financieras tradicionales han determinado estructuras de financiación débiles. Ello ha condicionado su potencial de actuación, y consecuentemente su significación económica y social.

De este perfil general se excluyen, relativamente, los casos excepcionales de las denominadas entidades singulares, ONCE, Cruz Roja y Cáritas, y otras macro-entidades que cuando no poseen una financiación muy privilegiada por parte de la administración, realizan actividades empresariales, o son nutridas merced a actividades de mecenazgo.

Este planteamiento se ha visto especialmente agudizado con el advenimiento y posterior permanencia de la crisis económica, que ha evidenciado el absoluto desvalimiento de las arcas públicas, la mayor reticencia del sector financiero al apoyo de entidades no lucrativas, y el repliegue de las donaciones privadas. De esta manera el sector no lucrativo se ha visto impelido a reorientar sus actividades de fundraising, tornando su mirada hacia la sociedad.

En este contexto se ha venido gestando una novísima forma de financiación social denominada crowdfunding. Aunque los primeros hitos históricos del crowdfunding hunden sus raíces en la Norteamérica del siglo XVII (la primera publicación financiada a través de esta modalidad fue The Penniless Pilgrimage, John Taylor, 1618), su especial y significativo desarrollo, merced a las nuevas tecnologías, viene dándose desde mitad de la pasada década.

En España, se trata de un fenómeno "neonato", apareciendo las primeras iniciativas en el 2010, y despegando con fuerza a partir del 2012. Se trata de una herramienta de fundraising, que está adquiriendo una significativa y retadora importancia de cara a la financiación social, y que ha comenzado a vertebrarse como actividad financiera alternativa merced a sus organizaciones asociativas. Así, en julio del 2013, se creó la Asociación Española del Crowdfunding, que ejerce un relevante papel de lobby en favor de una regulación del sector, apostando por una nueva ley de mecenazgo.
El crowdfunding (también llamado, financiación colectiva, en masa o micromecenazgo on-line) conceptualiza la práctica de las personas que hacen red para conseguir dinero u otros recursos y financiar un proyecto o propósito concreto. Su metodología sencilla y su origen y ubicación en la propia base social, hacen del crowdfunding un novedoso y relevante instrumento financiero para las entidades de economía social. Bajo distintas fórmulas operativas (recompensas, donaciones, préstamos, acciones) esta figura va adquiriendo un papel de primer orden a nivel mundial experimentando un ritmo de crecimiento superior al $200 \%$ anual.

El objeto de este trabajo es analizar el crowdfunding para la financiación de los proyectos sociales del sector no lucrativo, estudiando sus potencialidades, pero también sus retos como instrumento alternativo a la financiación tradicional (Sajardo, 2014b).

\section{Sobre el contexto financiero actual del sector no lucrativo}

En el presente contexto de crisis económica, generador de incertidumbre e inestabilidad social, la sociedad europea asiste, cuasi impasible, a la paulatina deconstrucción de su Estado de bienestar. Bajo la guía de políticas de corte neoliberal, se viene provocando una deriva desde lo público a lo privado, con consecuencias directas en el recorte de derechos sociales edificados desde la segunda mitad de la pasada centuria, que ha sido especialmente gravosa en España, por lo profundo y dilatado de la caída. En este contexto, el sector no lucrativo se ha visto impelido a asumir, progresivamente, el papel de actor principal en el mantenimiento del bienestar social (fundamentalmente materializado en ayudas de emergencia social) y, en no pocas ocasiones, en la ampliación de derechos y servicios a la ciudadanía, cubriendo los espacios que ha ido abandonando el sector público. La consecuencia directa ha sido un impacto negativo en sus estructuras financieras.

En España, desde el inicio de la crisis, se ha producido una reducción considerable en el presupuesto del conjunto de las comunidades autónomas para servicios sociales, en concreto a lo largo del cuatrienio 2009 al 2012 esta reducción ha sido de un 3,64\%, lo que representa un volumen global de más de 478 millones de euros. Ello ha supuesto en términos relativos, un decremento en el gasto por habitante de un $1,75 \%$ para el conjunto del Estado, de $€ 280,03$ en el 2009 a $€ 275,13$ en el 2012. De igual forma, se reduce 
el porcentaje que representa el gasto en servicios sociales en relación al PIB, pasando a nivel español del $1,25 \%$ en el 2009 al 1,21\% en el 2012, de esta manera se evidencia el claro retroceso del sector (García, Barriga, Ramírez y Santos, 2013). Al mismo tiempo, las necesidades reales se han visto incrementadas sustancialmente, a tenor del crecimiento de la pobreza y la exclusión social en España aumentando del 23,1\% en el 2008 hasta el 26,8\% en el 2012 (PTs, 2012).

El impacto de la crisis económica es manifiesto para el sector no lucrativo, de forma que ocho de cada diez entidades encuestadas en el 2010 reconocen dificultades de financiación, 20 puntos porcentuales más que en el 2008. Por ello, el principal reto que se plantean las entidades que lo conforman son los problemas de financiación (Equipo de Investigación Sociológica [EDIS], 2013). La financiación pública representó en el 2011 el 70\% de su presupuesto, en claro avance desde el 2008, año de inicio de la crisis, donde suponía el 64\% (PwC, 2014). Esta alta dependencia financiera pública contrasta con una disminución en la aportación de la filantropía privada tradicional, ${ }^{2}$ puesto que en el 2011, un $17,2 \%$ de los españoles habían finalizado, reducido o sustituido sus aportaciones a entidades no lucrativas (Adeco, 2011). El aporte financiero empresarial se ha elevado ligeramente desde el 2008 alrededor del 1\% (PWC, 2014).

Como consecuencia, por un lado, y a pesar de ello, por otro, el sector no lucrativo, se ha convertido en una pieza clave en el ámbito social, a tenor del número de entidades existentes, la gran variedad de programas que desarrollan, la masa voluntaria que es capaz de movilizar, el empleo que generan y el gran número de beneficiarios a los que atienden. ${ }^{3}$ Así, según el informe de PwC (2014), en el 2012, el tercer sector social español se ha nutrido con 8000 millones de euros de financiación, cuenta con 3 millones de

1 Durante el 2010 el Tercer Sector de Acción Social generó 47,6 millones de atenciones directas a personas, un 29,4\% más que en el 2007 (Anuario Tercer Sector de Acción Social, 2013).

2 En España, se estima un total de población donante del 10,3\%, frente al 33\% de media en Europa (AEFR, 2013).

3 En el 2010, el INE, en la Encuesta de Condiciones de Vida, introdujo el Indicador AROPE (At Risk Of Poverty and/or Exclusion). Se trata de un indicador agregado que pretende medir la pobreza y la exclusión social haciendo referencia al porcentaje de población que se encuentra en riesgo de pobreza y/o exclusión social. Además de contemplar las personas por debajo del umbral de la pobreza (únicamente en términos monetarios), el nuevo indicador aglutina otras relativas a la exclusión de la completa participación en la sociedad y del trabajo. socios, un $70 \%$ de sus recursos humanos son voluntarios, 400000 trabajadores remunerados y representa el $1 \%$ del PIB español. Todo ello en un contexto de alta dependencia financiera pública, en un país con necesidades sociales tendencialmente crecientes, como evidencia la bolsa potencial de más de 12 millones de personas en riesgo de pobreza y/o exclusión.

En el contexto de dependencia financiera pública, la situación del sector no lucrativo se hace adicionalmente más gravosa por dos hechos legislativos de potencial relevancia. De un lado, el continuo retraso en la actualización de la Ley de Mecenazgo y de otro, la aparición en marzo del 2014 de un borrador de modificación de la Ley de Subvenciones. Si el primer hecho perdura y el segundo se lleva adelante, podría significar la desaparición de más del 90\% de las entidades que componen el tercer sector social español (Plataforma oNG de Acción Social, 2012).

Para la plataforma que agrupa a los grandes actores del tercer sector ${ }^{4}$, la reforma de la Ley de Mecenazgo es considerada ineludible, imprescindible y urgente debido al contexto crítico actual y la necesaria basculación entre las fuentes de financiación del sector, que modifique sus porcentajes de participación hacia un mayor protagonismo de la financiación privada. No obstante, la tendencia ha sido a la inversa. Así, en el 2008, año de inicio de la crisis, el mix de financiación se dividía entre el 64\% para la financiación pública y el $36 \%$ para la privada; mientras que en el 2011 el peso específico de la financiación pública creció hasta un $70 \%$, si bien el monto global de la financiación recibida por el sector disminuyó un $2 \%$, reduciéndose al 30\% la financiación de origen privado (PWC, 2014).

La financiación privada al sector no lucrativo ha sido altamente reactiva a la crisis, puesto que han desaparecido financiadores históricos, se han mantenido estables, en niveles pretéritos, las bajas de socios, pero se ha producido una caída en el monto de nuevas altas. A ello se le adiciona la reestructuración del sector financiero que ha conllevado la desaparición de las cajas de ahorro como financiadores tradicionales del sector (PWC, 2014). En suma, un panorama

4 Las organizaciones del tercer sector que forman parte de esta iniciativa son la Asociación Española de Fundaciones (AEF), la Plataforma del Tercer Sector (POAS, PVE, EAPN-ES, Cermi, Cruz Roja Española, Cáritas y ONCE), la Asociación Española de Fundraising (AEFR), la Asociación de Fundaciones Andaluzas (AFA), la Asociación Extremeña de Fundaciones (AFEX), la Coordinadora Catalana de Fundaciones (CCF), la Coordinadora ONG para el Desarrollo (CONGDE) y la Plataforma de Infancia. 
de absoluta reticencia para la filantropía privada, que se hace especialmente significativo en el contexto de recortes presupuestales por parte de las administraciones públicas, si se desean mantener los niveles de atención existentes hasta el momento.

El sector no lucrativo presume la potencial reforma de la Ley de Mecenazgo como la posibilidad de que se impulse decisivamente la financiación privada en el sector. Esto es contemplado como una forma de fomentar la cohesión social, se logra una mayor participación privada en las actividades de interés general, y por consiguiente, se genera, fortalece y articula la sociedad civil. Para esto se plantea incrementar significativamente el porcentaje de deducción fiscal en el IRPF para las donaciones de personas físicas y, muy especialmente, establecer un límite por debajo del cual el 100\% del importe donado se desgrave directamente (AEFR, 2013).

Respecto al anteproyecto de modificación de la Ley de Subvenciones, la plataforma del tercer sector manifiesta una rotunda oposición, argumentando que esta no tiene en cuenta la naturaleza y singularidad específica del sector, lo que tendería a generar situaciones altamente críticas para las entidades que lo conforman. Entre los aspectos más negativos de la reforma debe destacarse el límite de la subvención al $75 \%$ del coste (art. 19), lo que conlleva a recurrir en un $25 \%$ a cofinanciadores privados (algo al alcance de pocas entidades), y el que los pagos a cuenta sean la excepción (art. 34), lo que obligaría a las entidades a recurrir al crédito. Adicionalmente, la reforma provocaría el aumento de la burocracia y de los trámites administrativos, tales como la no aceptación de facturas electrónicas como comprobante de pago, la ausencia de plazo por parte de la administración para el abono de la subvención, la necesidad de anticipar el importe íntegro de la subvención por parte de la entidad, o el hecho de que cualquier compra a terceros sea contemplada como una subcontratación.

Para la adecuación de la ley al sector, uno de los puntos clave que se manejan es la concepción de la entidad como beneficiaria de la subvención, cuando los beneficiarios de las acciones del sector no lucrativo son los más de 10 millones de personas objetivo de sus intervenciones. En este contexto, las entidades del sector ejercerían como meras correas transmisoras, sin posibilidad de obtener un retorno de sus acciones, por lo que se debería diferenciar entre la subvención de interés general de aquella que no lo es (Discapnet, 2014).

Un aspecto adicional para tener en cuenta en este contexto es el tratamiento que la Ley 27/2013, del
27 de diciembre, de racionalización y sostenibilidad de la administración local realiza de las competencias de las administraciones locales, que hasta el momento han jugado un papel clave en la distribución de las actividades de acción social y servicios sociales. Según el informe DEC (2013), la realidad confirma que:

Las Comunidades con mayor eficacia (mejores resultados) y eficiencia en servicios sociales (mejor relación entre el gasto que realizan y las prestaciones y servicios que ofrecen) son aquellas con mayor descentralización y mayor protagonismo local, mientras que las menos eficientes son las que menos protagonismo local tienen en sus servicios sociales. (García et al., 2013, p. 36).

El artículo 25 de la citada Ley 27/2013 reduce las competencias propias de las entidades locales en la materia de servicios sociales a informar y valorar, y a procurar atención inmediata a situaciones de exclusión. Lo que supone vaciarlas de todo el contenido prestacional que hasta ahora habían venido desarrollando, como las atenciones y servicios domiciliarios, residencias, viviendas tuteladas, servicios para la convivencia y la promoción de personas mayores, mujeres, niños y niñas, jóvenes, etc. Todos estos servicios pasan a ser considerados impropios de las entidades locales, y por lo tanto se las inhabilita para su libre prestación con base en criterios de política local (art. 74 Ley 27/2013).

El sumatorio de estos factores impele a cambios en el modo operativo del sector no lucrativo, muy especialmente en su ámbito financiero. En este sentido, la Plataforma del Tercer Sector, en la redacción del in Plan Estratégico del Tercer Sector de Acción Social, identifica un par de problemas principales a los que se enfrenta el Ts y a los que deberá encontrar solución en el periodo 2013-2016 para garantizar su adaptación adecuada a la nueva realidad política, económica y social. En primer término, enfatiza la insuficiencia de medios financieros ${ }^{5}$ para la prestación adecuada de bienes y servicios en un momento en el que se están produciendo recortes de derechos y en el que se están incrementando las necesidades sociales. En segundo lugar, señala el reducido peso del sector como actor social y político con la incidencia necesaria sobre la garantía de los derechos sociales.

\footnotetext{
Tales niveles de financiación solo podrían volver a alcanzarse a partir del año 2017 (PTS, 2012)
} 
En este contexto, el sector no lucrativo se torna impelido a reinventarse, a redescubrirse y a encontrar la vía hacia la innovación financiera. Se torna crucial la adopción de medios que vayan más allá de la mera donación desinteresada o filantropía, y que en cuya base de generación se introduzca la variable de los valores como un parámetro más a la hora de evaluar el retorno de la inversión. Este aspecto resulta imprescindible para elevar el ratio de donantes, que en España se halla en el 19\%, muy por debajo de la media europea del 33\% (PwC, 2014), y, adicionalmente, para incrementar el número de voluntarios, siendo la previsión para el 2016 de un 78\% de la plantilla voluntaria frente al 70\% existente en el 2011.

Una de las opciones financieras más relevantes para el sector no lucrativo en el momento actual se halla en la financiación en masa a través de Internet, denominada crowdfunding. Este instrumento financiero está alcanzando un relevante protagonismo en la actualidad, merced al surgimiento de un nuevo paradigma tecnológico que ha influido notablemente en el comportamiento de los ciudadanos, es un modelo de financiación que hunde sus raíces en el siglo XVII (Taylor, 1618).

\section{El crowdfunding o financiación en masa al sector no lucrativo: marco conceptual y realidad}

El término crowdfunding (financiación colectiva, financiación en masa o micromecenazgo online) identifica al fenómeno de las personas que hacen red para conseguir dinero u otros recursos y financiar un proyecto o propósito concreto. El crowdfunding permite a organizaciones e individuos conseguir recursos de una gran cantidad de pequeños aportantes comprometidos más que de unos pocos grandes (Asociación Europea de Crowdfunding, 2013).

El crowdfunding se articula bajo la idea de expandir la base de financiadores de un proyecto, en lugar de estrecharlo a una pequeña cantidad de los mismos; "permite financiar un proyecto o negocio logrando una gran cantidad de pequeñas aportaciones de recursos procedentes de un gran número de individuos" (Forbes, 2012). De esta manera se ubica en la base misma de la pirámide de la financiación, desdibujando su forma y convirtiéndola en un amplio rectángulo.

No se trata de un fenómeno nuevo, ya que hallamos su primer hito en 1618, cuando John Taylor logró el apoyo de 1650 individuos (suscriptores) para su publicación a cambio de una copia del libro, The Penniless Pilgrimage, una vez que este apareciese.

En fechas mucho más recientes encontramos el proyecto American Commitee of the Statue of Liberty, cuyo promotor Joseph Pulitzer, a través del periódico The New York World acopió \$101,091 para la financiación del pedestal de la Estatua de la Libertad. En los años ochenta lo encontramos con el nacimiento del software libre, en el marco del Proyecto GNU y de la Free Software Foundation de la mano de Richard Stallman.

La aparición de Internet permitió su extensión, resultando emblemática la iniciativa de la banda de rock progresivo británica Marillion, cuando ante la imposibilidad de realizar su gira en 1997 por falta de fondos, sus fans decidieron captar fondos por Internet, con un rotundo éxito (\$60 000). La gira se llevó a cabo, y se extendió la iniciativa. No obstante fue ArtistShare quien lanzó la primera plataforma para financiar los proyectos de artistas. Los primeros proyectos de Open Source (Wikipedia, Creative Commons, Mozilla) constituyeron su base de desarrollo, cuando los propios colaboradores ofrecían no solo su trabajo de forma desinteresada, si no también donaciones.

El crowdfunding incorpora una serie de principios y formas de trabajo que facilitan el acercamiento entre la sociedad y los proyectos sociales que apoya, como son:

1. La creación de una comunidad alrededor de los proyectos (frente a la comunidad alrededor de la organización que implican las donaciones tradicionales).

2. La lógica del crowdsourcing, o de multitudes inteligentes colaborando en pro de un proyecto social común.

3. El ciudadano asume un nuevo papel de participación activa en los proyectos, apareciendo la figura del prosumer.

4. Nuevos modelos de proyectos para nuevas formas de participación (voluntariado) y fundraising (financiación).

5. Unión entre las tradicionales y las más novedosas formas de participación: voluntariado, implicación, donación, activismo, redes...

Los sujetos implicados en un proyecto de crowdfunding son tres:

1. La entidad no lucrativa que busca financiación para su proyecto.

2. El colectivo de personas que creen en el proyecto y lo apoyan aportando fondos (aportantes o financiadores), a cambio de una recompensa inmaterial 
(de naturaleza psicológica), o tangible (no económica -descargas, copia libro o audiovisual, entradas al evento, camiseta, figurar en los créditos- o económica - retorno financiero: intereses, rentas, recuperar importe inversión-).

3. La plataforma tecnológica que facilita el contacto entre unos y otros, llevando a cabo las tareas de selección de proyectos, asesoramiento, licencia de software u otras por las que cobra comisión, oscilando generalmente entre el $5 \%$ y $9 \%$.

Atendiendo al objetivo que persigue el financiador del proyecto se identifican cuatro modelos de crowdfunding (The Crowdfunding Industry Report, 2012).

1. Modelo crowdfunding por acciones (Equity-based crowdfunding) en cuyo contexto los aportantes de fondos reciben compensación en forma de reparto de ingresos o beneficios. Por lo tanto, el objetivo para el financiador es el mero retorno financiero, constituyendo un sistema de inversión con opción a recibir participaciones o beneficios de la comercialización del output del proyecto. Este modelo ha adquirido un enorme potencial internacional a raíz de la aprobación en EE.UU. de la Jumpstart our Businesses Startups (Jовs) en el 2012.

2. Modelo crowdfunding por préstamos (lending-based crowdfunding), en el que los aportantes de fondos reciben ingresos periódicos, y esperan recuperar la inversión inicial, con pago de intereses o no.

3. Modelo crowdfunding por recompensas (reward-based crowdfunding), en el cual los colaboradores reciben recompensas no monetarias (algo simbólico, primera edición, entre otros).

4. Modelocrowdfundingpor donaciones(donation-based crowdfunding), merced al cual los aportantes poseen una motivación filantrópica, sin esperar nada tangible a cambio, solo les importa la causa que financian.

Por otro lado, y atendiendo a la gestión del resultado, se identifican dos modelos de plataformas de crowdfunding.

1. El modelo de "todo o nada", en cuyo contexto solo se cobra el dinero financiado si se llega al montante establecido, es decir, si el proyecto ha tenido éxito. Si el proyecto no ha alcanzado la meta financiera determinada, el dinero se devuelve a sus partícipes y el creador no recibe nada (sistema de pledges).

2. El modelo de "todo cuenta" en el que el creador del proyecto recibe la suma que ha logrado recaudar en el tiempo limitado, aunque no se haya alcanzado el resultado establecido como objetivo.

La mecánica operativa del crowdfunding es muy sencilla. La entidad no lucrativa confecciona la idea o proyecto a financiar, es decir, describe su objetivo sintéticamente ${ }^{6}$, indicando la cantidad necesaria para llevarlo a cabo, el tiempo de recaudación establecido, y el plan de recompensas que se quiere ofrecer a cada persona que aporte dinero según la cantidad que esta decide donar. Tras ello, lo envía a la plataforma web, la cual valora su idoneidad y acometimiento (crowdsourcing), aunque en algunos casos los proyectos son valorados de forma comunitaria.

Una vez valorado y aceptado para su lanzamiento, se publica en la plataforma de crowdfunding, comenzando entonces una profusa labor de difusión del proyecto y de recaudación. La entidad creadora del proyecto dispone de un plazo limitado (30, 60, 90 o 120 días) para acopiar la financiación, a lo largo del cual, junto con la plataforma difunden la idea a través de todos los medios posibles, dándole lo que se denomina viralidad.

Finalizado el plazo de recaudación establecido, si se llega al $100 \%$ de la financiación solicitada se considera que el proyecto ha sido exitoso. En este momento comienza un doble proceso. Por un lado, la entidad recibe el dinero al que se han comprometido los cofinanciadores, habitualmente menos la comisión establecida por la plataforma por su gestión; y de otro lado, aquella implementa el envío de recompensas a los aportantes, si las hubiese. Por lo general, si el proyecto no recibe el $100 \%$ de la financiación solicitada, el dinero recaudado hasta el momento no se cobra de las cuentas de cofinanciadores y no se realiza ninguna transferencia. El proceso concluye con un amplio apartado de agradecimientos de la entidad no lucrativa a los cofinanciadores.

Según el informe de The Crowdfunding Industry Report, (abril, 2012), a nivel global, el crowdfunding movilizó en el 2012 un volumen de \$2,7bn, con una tasa de crecimiento interanual (2012/2011) del 80\%, una tasa de éxito promedio del $40 \%$, y un tiempo promedio de logro del objetivo de 9,1 semanas. Las plataformas pioneras fueron las sajonas Indiegogo en el 2008, y Kickstarter, la cual apareció en el 2009. La tabla 1 sintetiza los principales datos del crowdfunding a nivel internacional, asimismo, la figura 1 expone el peso económico del crowdfunding a nivel internacional.

\footnotetext{
$6 \quad$ Frecuentemente se utilizan medios audiovisuales a través de la metodología del storytelling.
} 
Tabla 1

Las grandes cifras del crowdfunding a nivel mundial

\begin{tabular}{lcc}
\hline \multicolumn{1}{c}{ Norte América } & Europa & Países en desarrollo \\
\hline \$1,6 b (105\%) & \$945M (65\%) & 125\% \\
\hline \multicolumn{1}{c}{ REGIÓN } & N. ${ }^{\circ}$ PLATAFORMAS & \% / TOTAL \\
\hline Norte América & 208 & $45 \%$ \\
Europa & 186 & $40 \%$ \\
Oceanía & 18 & $4 \%$ \\
Latinoamérica & 27 & $6 \%$ \\
Asia & 18 & $4 \%$ \\
África & 4 & $1 \%$ \\
\hline
\end{tabular}

\begin{tabular}{lcc}
\hline \multicolumn{3}{c}{1.167 .000 campañas exitosas, 2011 } \\
\hline Norte América & Europa & Países en desarrollo \\
\hline 532000 & 654000 & 1000 \\
\hline \multicolumn{1}{c}{ Tipo Plataforma } & Tasa crecimiento 2012/2011 \\
\hline Donación & $41 \%$ \\
Recompensa & $79 \%$ \\
Préstamos & $50 \%$ \\
Acciones & $114 \%$ \\
\hline
\end{tabular}

Cuantías promedio solicitadas

MODELO DE PRÉSTAMOS

(Equity-based)

$21 \%>\$ 250.000$

MODELOS DONACIONES- RECOMPEN-

SAS $63 \%<\$ 5.000$

(Donation\&Reward-based)

\begin{tabular}{lc}
\hline \multicolumn{1}{c}{ Tipo Plataforma } & Tiempo promedio al objetivo (semanas) \\
\hline Donación & 10,2 \\
Recompensa & 10 \\
Préstamos & 4,8 \\
Acciones & 8,2 \\
\hline
\end{tabular}

Nota. Adaptado de The Crowdfunding Industry Report, por Crowdsourcing, LLC, 2012.

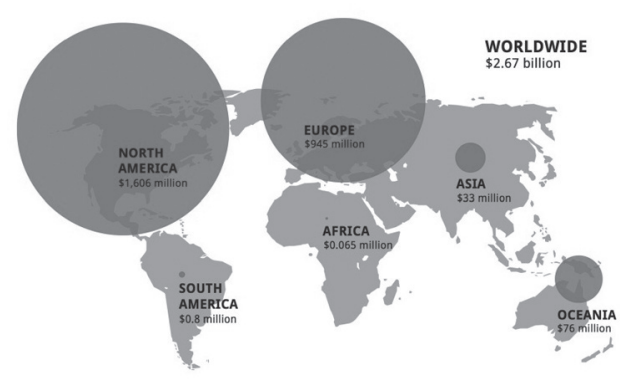

E. Total funds raised during 2012 in USD

Figura 1. Peso económico del crowdfunding a nivel internacional. Elaboración propia.
En España, el fenómeno del crowdfunding es de desarrollo muy novedoso, a partir del 2012, si bien las más primeras, y por el momento, más relevantes plataformas aparecieron poco tiempo antes, como el caso de Lanzanos, Verkami, o Goteo, todas del 2010. En el ámbito social las pioneras han sido migranodearena y miAportación en el 2010.

A tenor de los datos de la Asociación Española de Crowdfunding, en España existen 86 plataformas de temática general y no específicamente dedicadas a la financiación de proyectos sociales, cuyas principales cifras se exponen en la tabla 2:

Tabla 2

Plataformas de crowdfunding general en España

\begin{tabular}{cccc}
\hline Recompensas & Donaciones & Préstamos & Inversión \\
$52(59,52 \%)$ & $10(11,90 \%)$ & $6(7,1 \%)$ & $18(21,42 \%)$ \\
\hline
\end{tabular}

Nota. Elaboración propia.

La tabla 3 muestra la comparativa de las plataformas españolas principales y pioneras con una de las más relevantes a nivel internacional, Kickstarter.

En el caso concreto de plataformas dedicadas a la financiación de proyectos sociales se localizan un total de 24, mayoritariamente de tipo donaciones (véase tabla 4 ).

Por último, y sin ánimo de exhaustividad, y con el mero objeto de presentar una pequeña muestra de la realidad del crowdfunding para la financiación de los proyectos e iniciativas del sector no lucrativo, se recogen dos ejemplos, uno a nivel internacional, y otro desarrollado en España, en concreto en la Comunidad Valenciana. El primer caso surge de la organización Avaaz, ${ }^{7}$ cuando en septiembre del 2013, lanzó una ronda de crowdfunding, a través de su red de ciberactivistas, para la consecución de fondos dirigidos al restablecimiento de las escuelas, y evitar así que los niños y niñas víctimas en el conflicto armado de Siria perdieran las oportunidades en su vida. En menos de una semana, con pequeñas contribuciones de 49.514 personas, provenientes de 143 países diferentes, Avaaz logró reunir la cantidad de un millón de dólares americanos.

El que fuera primer ministro del Reino Unido, Gordon Brown, como miembro del comité encargado de la gestión de estos fondos, escribió en The

$7 \quad$ Para más información consulte www.avaaz.org 
Tabla 3

Comparativa principales plataformas de crowdfunding españolas

\begin{tabular}{lcccccccc}
\hline & Año inicio & Media proyectos mes & Media de coste & $\begin{array}{c}\text { Ratio } \\
\text { Éxito }\end{array}$ & Comisión & $\begin{array}{c}\text { Media } \\
\text { donación }\end{array}$ & Product & Naci. \\
\hline Goteo & 2010 & 8 & 5000 & $55 \%$ & $8 \%$ & $€ 50$ & MyE & ESP \\
Verkami & 2010 & 100 & 3700 & $73 \%$ & $5 \%$ & $€ 25$ & MyA & ESP \\
Kickstarter & 2009 & 2200 & 4500 & $44 \%$ & $5 \%+5 \% A m z$ & $€ 25 / € 20$ & MyC & USA \\
Lánzanos & 2010 & 50 & 3000 & $60 \%$ & $5 \%-1 \%$ PS & $€ 20$ & C,A,L,R & ESP \\
\hline
\end{tabular}

Nota. Tomado de X-Net (2013).

Tabla 4

Tipología plataformas de crowdfunding social en España

\begin{tabular}{ccc}
\hline Recompensa & Donación & Préstamos \\
$5(24,8 \%)$ & $18(75 \%)$ & $1(4,16 \%)$ \\
\hline
\end{tabular}

Nota. Elaboración propia.

Independent ${ }^{8}$ el 22 de septiembre del 2013 "para lograr esto, necesitamos que la comunidad internacional muestre una respuesta coordinada a esta crisis sin precedentes". El crowdfunding ha sido la herramienta que ha proporcionado la velocidad y la difusión necesarias para convertir en realidad este proyecto.

Un segundo ejemplo es el desarrollado, a una escala mucho más local, por Escola Valenciana (Ev), entidad que agrupa a 29 asociaciones y entidades educativas de ámbito comarcal y regional. ev lanzó, a través de la plataforma de crowdfunding Verkami, ${ }^{9}$ una ronda de financiación con el objetivo inicial de obtener los fondos suficientes para poder cubrir sus costes operativos hasta final de año y, una vez alcanzado este objetivo, obtener nuevos donantes recurrentes de pequeños importes, con el ánimo de conseguir la autosuficiencia económica (ver tabla 5).

En esta ronda de crowdfunding, Ev alcanzó su objetivo, €60000, gracias a la aportación de 1623 donantes. La difusión de esta iniciativa se realizó a través de la página de Facebook ${ }^{10}$ que utiliza Ev para sus estrategias comunicativas, que cuenta con 7285 fans o seguidores, y de su cuenta en Twitter ${ }^{11}$ a la que siguen 11615 usuarios.

\footnotetext{
8 Véase http://www.independent.co.uk/voices/comment/gordonbrown-a-little-learning-could-provide-an-awful-lot-of-help-8833063. html

9 Véase http://www.verkami.com/projects/6767-fes-escola-valenciana

10 Véase www.facebook.com/escolavalenciana

11 Véase twitter.com/escolatv
}

Tabla 5

Importes de donación y número aportantes en el crowdfunding de $E V$

\begin{tabular}{cc}
\hline Importe Donación & Número de Donantes \\
\hline$€ 10$ & 257 \\
$€ 15$ & 60 \\
$€ 20$ & 599 \\
$€ 300$ & 19 \\
$€ 500$ & 6 \\
$€ 1000$ & 3 \\
\hline
\end{tabular}

Nota. Elaboración propia.

\section{El potencial del crowdfunding social en España}

Con el objeto de evidenciar el potencial financiero del crowdfunding social para el sector no lucrativo español se utilizaron dos estudios nacionales relevantes, el primero de la Asociación Española de Fundrainsing $(2013)^{12}$, relativo al perfil del donante español, y el segundo de Iwith.org, realizado por Two Much Studio y Net Quest (2013).

El primer estudio se basó en una metodología CATI, mediante la realización de 1756 entrevistas a personas de ambos sexos de 18 o más años, de todo el territorio español, durante el mes de octubre del 2012.

12 El estudio ha sido elaborado por la consultora Salvetti \& Llombar, se ha realizado sobre una muestra de 1.756 entrevistas en el sizing y 1.674 en el estudio del perfil del donante (error muestral del $+2,3$ y $+2,4 \%$ respectivamente, para $\mathrm{P}=\mathrm{Q}=50 \%$ con un $95 \%$ de intervalo de confianza). 
Tabla 6

Potencial donantes en España, 2012

\begin{tabular}{lc}
\hline \multicolumn{1}{c}{ Tipo donante } & Magnitud (\%) \\
\hline Nunca ha donado & 43,0 \\
Ha donado alguna vez & 26,3 \\
No, pero sí en los próximos meses & 11,3 \\
Sí, en los últimos 12 meses & 19,4 \\
\hline
\end{tabular}

Nota. Tomado de El perfil del donante en España-Resumen de Prensa, por Asociación Española de Fundraising (AEFR), 2013.
A tenor de la tabla 6 se calcula un total de 7500765 individuos donantes reales en España, el $19,4 \%{ }^{13}$ de la población ${ }^{14}$, y 4383996 donantes potenciales, es decir, aquellos que habiendo sido o no previamente donantes, desean hacerlo próximamente. El importe medio por donación alcanza los de 164 euros, aunque los porcentajes de mayor incidencia son aquellas donaciones de más de 250 euros (21\%), entre $30-60$ euros (16\%), y entre 90-120 euros (16\%).

La tabla 7 muestra el perfil del donante en función de variables sociodemográficas, personales y tecnológicas, este último de crucial importancia en el tema del crowdfunding. De él pueden inferirse las retadoras posibilidades del crowdfunding social en España, en la medida en que el $99 \%$ de los donantes usan Internet, y el 91\% tienen teléfono móvil.

Tabla 7

Perfiles del donante en España, 2012

\begin{tabular}{|c|c|c|}
\hline Perfil Sociodemográfico & Perfil Personal e Ideales (\% individuos) & Perfil Tecnológico \\
\hline $\begin{array}{l}\text { - Tienen una edad media de } 46,9 \text { años } \\
\text { - Hombres } 54 \% \\
\text { - Mujeres } 46 \% \\
\text { - Personas casadas o que viven en pareja } 65 \% \\
\text { - Con hijos } 49 \% \\
\text { - Estudios universitarios } 66 \% \\
\text { Actividad profesional: } \\
\text { - Mandos intermedios } 24 \% \\
\text { - Jubilado/pensionista } 15 \% \\
\text { - Profesional liberal } 14 \%\end{array}$ & $\begin{array}{l}\text { - Me defino como una persona más de } \\
\text { izquierdas } 58 \% \\
\text { - Me declaro creyente } 63 \% \\
\text { - Me considero una persona realista } 68 \% \\
\text { - Me considero una persona racional } 53 \% \\
\text { - Reciclo } 90 \% \\
\text { - Puedo contribuir en la conservación } \\
\text { medioambiental } 86 \%\end{array}$ & $\begin{array}{l}\text { 99\% Usa Internet } \\
91 \% \text { Tiene móvil } \\
\text {-Utiliza las webs de las ONG para estar en } \\
\text { Contacto/informarse de ellas Sí } 59 \% \\
\text {-Estaría dispuesta a realizar donaciones } \\
\text { online Sí } 44 \% \\
\text { - Le gustaría recibir información por e-mail } \\
\text { de la ONG Sí } 47 \% \\
\text {-Haría donaciones a través del móvil Sí 10\% } \\
\text {-Le gustaría recibir información por sMsL de } \\
\text { la oNG Sí } 11 \%\end{array}$ \\
\hline
\end{tabular}

Nota. Tomado de El perfil del donante en España - Resumen de Prensa, Asociación Española de Fundraising (AEFR), 2013.

Tabla 8

Utilización de redes sociales por los donantes a entidades sociales

\begin{tabular}{|c|c|c|c|c|c|c|c|c|}
\hline & $\begin{array}{c}\text { Tienen } \\
\text { Perfil }\end{array}$ & Lo usan & $\begin{array}{c}\text { Es amigo/fan } \\
\text { en esta red } \\
\text { social }\end{array}$ & $\begin{array}{c}\text { Dispuesto a ser } \\
\text { amigo/fan en } \\
\text { esta red social }\end{array}$ & $\begin{array}{l}\text { Se informa está } \\
\text { en contacto con } \\
\text { alguna ONG }\end{array}$ & $\begin{array}{c}\text { Gustaría } \\
\text { recibir info de } \\
\text { ONG }\end{array}$ & $\begin{array}{l}\text { Ha participa- } \\
\text { do en alguna } \\
\text { iniciativa de } \\
\text { alguna ONG }\end{array}$ & $\begin{array}{c}\text { Participaría en } \\
\text { alguna inicia- } \\
\text { tiva en esta red } \\
\text { social }\end{array}$ \\
\hline Facebook & $53 \%$ & $48 \%$ & $12 \%$ & $15 \%$ & $12 \%$ & $18 \%$ & $9 \%$ & $22 \%$ \\
\hline Twitter & $19 \%$ & $13 \%$ & $5 \%$ & $5 \%$ & $5 \%$ & $1 \%$ & $3 \%$ & $7 \%$ \\
\hline Tuenti & $6 \%$ & $4 \%$ & $1 \%$ & $1 \%$ & $1 \%$ & NA & $0 \%$ & $1 \%$ \\
\hline Instagram & $3 \%$ & $2 \%$ & $0 \%$ & $1 \%$ & $0 \%$ & NA & $0 \%$ & $1 \%$ \\
\hline LinkedIn & $3 \%$ & $3 \%$ & $0 \%$ & $1 \%$ & $0 \%$ & NA & $0 \%$ & $1 \%$ \\
\hline Ninguna & $41 \%$ & $46 \%$ & $83 \%$ & $79 \%$ & $84 \%$ & $80 \%$ & $89 \%$ & $74 \%$ \\
\hline
\end{tabular}

Nota. Tomado de El perfil del donante en España - Resumen de Prensa, Asociación Española de Fundraising (AEFR), 2013.

13 Cifra alejada de la media europea sita en el 33\%. 
Respecto a los porcentajes relativos a la participación en redes sociales, en la tabla 8 se observan los comportamientos en el perfil del donante, todo lo cual enfatiza el potencial del crowdfunding español.

En segundo lugar, se considera un estudio que fue realizado a lo largo de septiembre del 2011, a través de Internet. La muestra fue de 1184 individuos mayores de 16 años sobre una población española de 20 millones. Los datos indican que el 26,6\% de los españoles son potencialmente permeables a las donaciones directas aunque no lo hayan hecho nunca, siendo el apoyo puntual a proyectos concretos la fórmula más valorada, con el 40,8\%. De la unión de ambos datos se establece un potencial de 5,4 millones de personas en España permeables a las aportaciones directas en dinero, y por lo tanto al crowdfunding, si el proyecto les genera el suficiente interés y confianza, y aunque no lo hayan hecho nunca (véase tabla 9).

Tabla 9

Tipo de apoyo preferido por el donante

\begin{tabular}{lc}
\hline \multicolumn{1}{c}{ Tipo de apoyo } & Porcentaje \\
\hline Apoyo directo a iniciativas puntuales concretas & $40,8 \%$ \\
Ingreso cuotas periódicas & $11,1 \%$ \\
Ingreso mensual de una micro-cuota & $9,8 \%$ \\
Las tres son igualmente apropiadas para mí & $15,4 \%$ \\
Ninguna alternativa es apropiada para mí & $12,7 \%$ \\
NS/NC & $10,1 \%$ \\
TOTAL & $100 \%$ \\
\hline
\end{tabular}

Nota. Elaboración propia.

\section{Luces y sombras del crowdfunding como vector financiero del sector no lucrativo}

El crowdfunding genera a las entidades del sector no lucrativo un efecto económico netamente positivo al facilitar la obtención de fondos para financiar sus actividades o proyectos. Evita la dependencia financiera de unos pocos donantes, y el recurso a los mercados de financiación tradicional.

A través de las nuevas tecnologías, se genera la posibilidad de que grandes masas de personas confíen en un proyecto o idea, más allá de las redes personales y profesionales de la entidad y de sus gestores ${ }^{15}$. Constituye una manera relativamente fácil, y sin coste económico añadido, de publicitar las actividades del sector no lucrativo, y por lo tanto, una vía clara para mostrar su amplitud, dinamicidad, y vocación-misión social. Asimismo, permite una mayor cohesión con la sociedad, la cual interactúa positivamente con las entidades, aportando, en un primer momento, tanto su opinión en el diseño de los proyectos a financiar ${ }^{16}$ como su neto apoyo al financiarlos posteriormente. Los donantes, amén de recibir unas recompensas materiales simbólicas como agradecimiento por su colaboración, obtienen un valor añadido adicional como es la recompensa psicológica que produce el colaborar en proyectos sociales.

No obstante, la figura del crowdfunding en el momento actual de desarrollo se enfrenta a una serie de aspectos críticos o retos a los que debe hacer frente, si se pretende erigir como una opción financiera viable y solvente para el sector no lucrativo.

El primero, es la denominada escalabilidad. El análisis práctico de esta herramienta muestra que es útil para financiar proyectos de presupuesto bajo o medio, y para un tiempo reducido, de manera que resulta complicada para proyectos que requieran grandes inversiones, como vía financiera a largo plazo, o como fuente única o básica de financiación. Como ejemplo ilustrativo pueden mostrarse los datos que arroja la plataforma pionera Kickstarter, y que se recogen en la tabla 10, relativos al nivel de éxito de los proyectos financiados en función de su presupuesto objetivo.

Tabla 10

Incidencia de proyectos exitosos según niveles de objetivo (2011)

\begin{tabular}{lc}
\hline Proyectos objetivo 1000-10 000\$ & $58,8 \%$ \\
Proyectos objetivo < $1000 \$$ & $11,8 \%$ \\
Proyectos objetivo $10000-20000 \$$ & $11,8 \%$ \\
Proyectos objetivo $100000-1 \mathrm{M} \$$ & $0,8 \%$ \\
Proyectos objetivo > 1M\$ & $0,03 \%$ \\
\hline
\end{tabular}

Nota. Tomado de http://www.kickstarter.com.

15 En otras palabras, mediante el proceso de "boca a boca" (word of mouth) se genera el efecto "bola de nieve" (snowball effect).

16 Prácticamente la totalidad de las plataformas de crowdfunding realizan un estudio de idoneidad de los proyectos antes de ser lanzados, pero una parte de las mismas permiten recabar la opinión de los potenciales financiadores de proyectos, coadyuvando, a través de una corriente de de feedback informativo, en su diseño y estructura. Este es el caso de las plataformas españolas Lanzanos o Verkami. 
La principal conclusión que puede derivarse para el contexto español, y que es constatada por la Asociación Española de Crowdfunding (SCF), estriba en que sin cambios legislativos relevantes ${ }^{17}$, esta herramienta solo tendría sentido para proyectos de entre 3000 a 15000 euros, siendo necesario un tiempo dilatado para su éxito, y una gran visibilidad mediática para cruzar ciertos niveles de financiación.

En segundo lugar, ha de indicarse que los fondos captados a través de crowdfunding son procedentes fundamentalmente de rentas del trabajo, dado que al no ser considerado, desde la perspectiva del aportante, como una inversión sino como una colaboración, en pocas ocasiones se derivan fondos provenientes del capital financiero o fondos de inversión. Esto tiene el efecto de limitar el espectro de caudales al que pueden acceder los proyectos, y por lo tanto, el tamaño y el volumen de proyectos, así como su posible realización periódica.

En tercer lugar, se ha de evidenciar el agotamiento de las comunidades de potenciales financiadores. Así, se constata que el éxito de una campaña de crowdfunding se halla altamente vinculado a la viralidad de la entidad promotora, es decir, a su capacidad de movilización en comunidades cercanas y en grupos sociales beneficiarios. De esta manera, cuanto más grande sea el tamaño de aquellas, mayor será su potencial, y a la inversa, cuanto más sectorial o específico sea el proyecto, y por lo tanto su tamaño, menor será su grado de éxito. La solución a este problema pasaría por regular la posibilidad de que las plataformas recibieran fondos de capital financiero (X-Net, 2013).

Se da asimismo una alta polarización de iniciativas a financiar. Por lo general existe una mayor popularidad en proyectos musicales, audiovisuales, y publicaciones o similares. Aquellos proyectos arriesgados o críticos cuestan más para financiar, siendo en algunos casos marginados. Existe una dominancia de proyectos de base material frente a efímeros o intangibles, campo objetivo del sector no lucrativo. Las comunidades con escaso acceso a redes (marginados, mayores, analfabetos digitalmente) encuentran una mayor dificultad para su implementación.

17 El Anteproyecto de Ley de Fomento de la Financiación Empresarial que incluye en su Título v el Régimen Jurídico de las Plataformas de Financiación Participativa, dedicado, de manera muy restrictiva, a regular el crowdfunding de participaciones en proyectos empresariales (equity crowdfunding) y el de préstamos (crowdlending), quedando excluido todo tipo de regulación respecto a donaciones y recompensas, de absoluta idoneidad para la financiación del sector no lucrativo.
Resulta por tanto necesaria la generación de redes específicas para llegar a nichos de población con menor presencia en redes, y de sistemas que mitiguen o erradiquen estas formas de desigualdad.

A nivel operativo, deben considerarse tres aspectos. De un lado, dado que la dinámica de funcionamiento del crowdfunding se basa en que la entidad no lucrativa explique su idea o proyecto en una plataforma, pueden darse plagios o copias del mismo, en desmedro de su potencial financiador. De otro lado, existe el riesgo de generar un reducido feedback por parte de los financiadores, en relación con los inversores tradicionales (caso de banca tradicional), con mayor capacidad de aporte crítico inmediato al proyecto a financiar. Asimismo, deben considerarse los costes asociados, pues el crowdfunding es una herramienta de bajo coste económico, pero no nulo. Así, junto con los costes de gestión que implica el uso de la plataforma (entre un 5-9\%), deben unirse las tasas bancarias por transferencias o alternativamente los costes de las pasarelas de pago, esto sin contar el coste del tiempo invertido en la gestión de la campaña de crowdfunding por parte de la entidad no lucrativa ${ }^{18}$.

Debe argumentarse, adicionalmente, que en el momento actual el sector se halla inmerso en una enorme inseguridad jurídica debido a la falta de un marco regulatorio específico, amén de la pretendida regulación de dos de sus modalidades (préstamos e inversión), en el seno del mencionado anteproyecto de Ley de Financiación Empresarial. Resta por tanto la legislación sobre el funcionamiento del crowdfunding social basado en donaciones o recompensas, campo específico de la operativa del sector no lucrativo, y que encajaría de lleno en el seno de una nueva Ley de Mecenazgo. Todo este vacío legal se ha paliado con el trabajo individual de las plataformas, generándose incoherencias, inseguridades o dudas de operación.

En este contexto, un grupo de plataformas de crowdfunding ha iniciado una campaña para que la

18 Se estima que los costes asociados a una campaña de crowdfunding pueden alcanzar el $30 \%$ del presupuesto estimado (X-Net, 2013). El sumatorio lo conformarían las comisiones (5\%9\%), más las tasas bancarias por el coste de las transferencias, o las pasarelas de pago como Paypal que cobran a su vez si consideran que ha existido compra $(3,4 \%+€ 0,35$ entre 0 - €2500). Además si quienes reciben el dinero final son empresas o autónomos han de facturar para obtener el ingreso (21\% IVA). Junto a ello, se deben incluir los costes de producir y distribuir las recompensas y posibles bonificaciones, y las horas de trabajo invertidas al día por parte de la entidad para su gestión (una media de cuatro horas al día para proyectos de 12000 euros). 
financiación colectiva de proyectos se regule y pueda crear empleos de calidad. El objeto de esta campaña es solicitar al Congreso, a la Comisión de Cultura encargada de la Ley de Mecenazgo, y la Ley del Emprendedor a través de varios ministerios, una serie de propuestas concretas, que se detallan en la tabla 11.

Tabla 11

Propuestas de regulación en apoyo del crowdfunding

\begin{tabular}{|c|c|}
\hline $\begin{array}{l}\text { 1. Incremento de las deduccio- } \\
\text { nes de aportaciones a ENLS } \\
\text { 2. Flexibilizar los requisitos } \\
\text { de declaración de Utilidad } \\
\text { Pública, para que el mecenas } \\
\text { pueda acogerse a las deduc- } \\
\text { ciones. } \\
\text { 3. Reducción del Iva entre } \\
\text { el } 4 \% \text { y } 30 \% \text { para proyectos } \\
\text { sociales, culturales, educativos, } \\
\text { científicos, deportivos, investi- } \\
\text { gación y desarrollo. } \\
\text { 4. Posibilidad de que cualquier } \\
\text { persona pueda participar en } \\
\text { cualquier empresa mediante } \\
\text { pequeñas donaciones. }\end{array}$ & $\begin{array}{l}\text { 5. Desgravación fiscal de } \\
\text { las pequeñas aportaciones } \\
\text { de inversores que apoyen a } \\
\text { empresas. } \\
\text { 6. Reducción del porcentaje a } \\
\text { pagar por los intereses o bene- } \\
\text { ficios de sus aportaciones } \\
\text { 7. Priorizar o subvencionar } \\
\text { proyectos vía crowdfunding. } \\
\text { 8. Políticas activas de difusión } \\
\text { del crowdfunding. } \\
\text { 9. Promocionar plataformas } \\
\text { actuales o futuras. }\end{array}$ \\
\hline
\end{tabular}

Nota. Tomado de www.yoapoyoelcrowdfunding.com.

En este contexto, el papel del sector público deviene crucial. Así, resulta básica una regulación específica del sector, que sea comprensiva de la idiosincrasia interna que implica. Y en este sentido se deberían apoyar las plataformas que propicien un retorno social en pro del bien común, que fomenten la transparencia y el desglose económico de los proyectos desarrollados por las plataformas, y que fortalezcan los principios democráticos, transparentes, generadores de modelos éticos y sostenibles. Asimismo la administración debería contribuir al crecimiento de las plataformas, invirtiendo en su consolidación (y no en los proyectos que albergan), en la reforma de sus estructuras técnicas que eleven su eficiencia, e instrumentar medidas concretas que vehiculicen el punto de encuentro entre donantes-financiadores y entidades, sobre la base de una amplia seguridad jurídica para ambas partes.

Por último, la consolidación del crowdfunding como herramienta de financiación fundamental de proyectos sociales desarrollados por el sector no lucrativo, no debe basarse en la búsqueda de la mera donación por parte del aportante, si no arriesga el convertirse en un instrumento puntual, ad hoc, altamente aleatorio, y por lo tanto de reducido potencial. Su utilización debe plantearse desde una perspectiva más amplia, desde la perspectiva de la sociedad mecenas, aquella que busca un retorno social permanente de y con su aportación. Aquella que se moviliza con base en los valores comunes y colectivos de la solidaridad, la cooperación, la heteroayuda (abanderados por la economía social y el sector no lucrativo), pero que es consciente de la individualidad (en el sentido de unicidad), la libertad de acción, pero con autoresponsabilidad social y la autonomía de su aportación. El crowdfunding hundiría así sus raíces en la generación de un nuevo planteamiento social del altruismo y la filantropía que podría estar basado en el contradictorio término del "individualismo colectivo" (Sajardo, 2014b), y que estaría altamente vinculado a la perspectiva del valor social y a las novísimas ideas de la motivación intrínseca (Pink, 2009).

\section{Conclusiones}

En España, el persistente marasmo económico actual ubica al sector no lucrativo en una posición delicada a nivel financiero. Su tradicional dependencia de la ayuda financiera externa se hace especialmente cruda ante la ingente reducción de la ayuda pública, al receso de los aportes empresariales, que acusan números rojos en sus cuentas de resultados, y de los donantes privados, sobreviviendo impasibles ante un contexto de progresivo desmantelamiento del Estado social. En este contexto, el sector no lucrativo se halla en la doble encrucijada, de un lado, de tener que satisfacer necesidades sociales antes cubiertas públicamente, con el consiguiente repliegue del Estado; y de otro, el reducido margen de actuación que prestan sus estructuras financieras altamente debilitadas. La situación ha llevado a las principales entidades representativas del sector a dibujar un panorama de corto plazo especialmente negativo para el mantenimiento del sector, que en el límite arriesgaría, incluso, su supervivencia.

En este contexto, las nuevas herramientas de comunicación y relación social que han aparecido con el nuevo paradigma tecnológico, surgido en los inicios del siglo XxI, dota al sector no lucrativo de instrumentos aportadores de capacidades con las que no contaban anteriormente ofreciendo oportunidades en numerosos ámbitos organizativos, que sin duda deben aprovechar, sobretodo en el tema financiero, lo que es particularmente relevante, cuando los principios sobre los que se asientan las nuevas tecnologías, como su naturaleza de red distribuida, la relevancia del principio de colaboración y de compartir, o su estructura democrática hallan un alto paralelismo con 
los principios de organización y de funcionamiento de la economía social.

La nueva sociedad plurinterconectada, plurinformada y multinivelada merced a la web 2.0 , se revela globalizada y sensibilizada en pro de los ámbitos sociales, y genera nuevas herramientas de financiación para el sector, entre ellas la nueva filantropía, la denominada financiación en masa on-line o crowdfunding. Esta nueva filantropía pone a disposición de las entidades no lucrativas la construcción de un conjunto de relaciones de mayor profundidad con los donantes que llegan a comprometerse profundamente con las causas que apoyan. Las entidades no lucrativas pueden ubicarse en este nuevo esquema como participantes en redes más complejas que solucionan necesidades, a través de una financiación más plural, menos dependiente y más sostenible.

El potencial del crowdfunding a nivel internacional se halla ampliamente constatado con tasas de crecimiento interanual del $200 \%$ para algunas plataformas. En España las posibilidades de esta nueva filantropía son especialmente retadoras, cuando el $99 \%$ de la población donante real y potencial usa Internet y el $91 \%$ tiene teléfono móvil, estimándose en 5,4 millones de personas el colectivo potencial de crowdfunding español. No obstante, para su correcta movilización, resulta, cuanto menos, necesario tener en cuenta un par de aspectos.

Primero, debe desarrollarse una regulación eficaz del sector, a tres niveles: de un lado, diferenciando adecuadamente entre sus usos para la entidad o empresa generadora del proyecto (causas sociales, emprendimiento, pre-venta), y sus tipologías consecuentes para el financiador o aportante (como donaciones, como préstamos, o como inversión), estableciendo en uno u otro caso su operativa; de otro lado, estimulando su desarrollo a través de incentivos fiscales para los donantes-cofinanciadores, bien en el seno de una ley de mecenazgo, bien en el ámbito de una regulación específica del sector. Adicionalmente, se debería regular la posibilidad de que las plataformas recibieran fondos de capital financiero, para evitar el agotamiento de las comunidades, cuyas aportaciones-donaciones se nutren principalmente de rentas del trabajo.

Segundo, debe fomentarse y delimitarse claramente su naturaleza y ámbito de actuación, para soslayar potenciales rivalidades con la financiación tradicional, por lo disruptivo que este modelo implica, pero sobre todo, no debe ser óbice para que la administración, bajo el velo de una mayor consolidación ciudadana, repliegue las responsabilidades sociales que la conquista social histórica ha ido edificando progresivamente y materializando a través de los Estados de bienestar.

\section{Referencias}

Adecco. (2011). Annual Report 2011. Adecco Group Company. Recuperado de http://ar.adecco.com/?id=ar2011

Asociación Española de Fundraising. [AEFR]. (2011). II Estudio de benchmarking sobre la situación de la captación de fondos y la rendición de cuentas en las ENL en España. Madrid: Asociación Española de Fundraising. Recuperado de http://www.plataformavoluntariado. org/ARCHIVO/documentos/recursos/Estudio_Benchmarking.pdf

Asociación Española de Fundraising. [AEFR]. (2013). El perfil del donante en España - Resumen de Prensa. Madrid: Asociación Española de Fundraising. Recuperado de http://aefundraising.org/upload/90/12/Estudio_perfil_donante_2012-_Resumenprensa.pdf

Asociación Europea de Crowdfunding (2013). Recuperado de http://crowdfunding.is/

Crowdsourcing, LLC. (2012). The Crowdfunding Industriy Report: Market Trends, Composition and Crowdfunding Platforms. Disponible en http://www.crowdfunding. nl/wp-content/uploads/2012/05/92834651-Massolution-abridged-Crowd-Funding-Industry-Report1.pdf

Equipo de Investigación Sociológica. [EDIS]. (2013). Anuario del Tercer Sector de Acción Social en España 2012. España: Fundación Luis Vives. Disponible en http:// www.luisvivesces.org/upload/95/52/2012_anuario_ tsas.pdf

García, G., Barriga, L., Ramírez, J. M. y Santos, J. (2013). Indice de desarrollo de los servicios sociales. Asociación Estatal de Directores y Gerentes en Servicios Sociales. Recuperado de http://www.directoressociales.com/ images/documentos/idec\%2007.06.2013.pdf

Plataforma de ONG de Acción Social. (2013). II Plan estratégico del Tercer Sector de Acción Social 2013-2016. Recuperado de http://www.plataformaong.org/ planestrategico/ARCHIVO/documentos/14/iipetsas.pdf

Pink, D. (2009). Drive: The Surprinsing Truth Abouth What Motivates US. USA: Riverhead Books.

PWC.(2014).Lareaccióndeltercersectoralentornodecrisis,ESADEObraSocial La Caixa. Recuperado dehttp://itemsweb.esade.es/00EC7794-111C-44C6-B27A-9EBFCB1A1D41/ FinalDownload/DownloadId-9687F74573550C709B0053AE32D19C05/00EC7794-111C-44C6-B27A-9EBFCB1A1D41/ 
wi/research/iis/publicacions/2014_Informe\%20tercer\%20sector.pdf

Sajardo, A. (2014b). Informe sobre el crowfunding social en España. Madrid: Fundación Manantial.

Sajardo, A., Serra Yoldi, I. (2007). Valoración económica del Voluntariado en la Comunidad Valenciana. Fundación de la Solidaridad y el Voluntariado de la Comunidad
Valenciana, Tirant lo Blanch. Recuperado de https:// www.academia.edu/1749330/Voluntariado_Valoracion_economica_del_voluntariado_Voluntariado_ corporativo

Taylor, J. (1618). The Peniless Pilgrimage. London: Edw Allde. Recuperado de https://archive.org/details/pennylespilgrim00taylgoog 